\title{
Relationship between the source size at diffuser plane and longitudinal spatial coherence function of optical coherence microscopy system
}

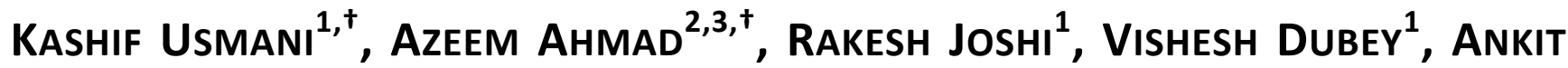 \\ BUTOLA ${ }^{1}$, DALIP SINGH MEHTA ${ }^{1,4}$
}

\author{
${ }^{1}$ Bio-photonics and Green Photonics Laboratory, Department of Physics, Indian Institute of Technology Delhi, New Delhi 110016, India \\ ${ }^{2}$ Department of Physics and Technology, UiT The Arctic University of Norway, Troms $\varnothing$ 9037, Norway \\ *Corresponding author: ${ }^{3}$ ahmadazeem870@gmail.com, ${ }^{4}$ mehtads@physics.iitd.ac.in \\ tThese authors contributed equally to this work.
}

\begin{abstract}
Coherence properties of light sources are indispensable for optical coherence microscopy/tomography $(\mathrm{OCM} / \mathrm{T})$ as they greatly influence the signal to noise ratio, axial resolution, and penetration depth of the system. In the present paper, we report the investigation of longitudinal spatial coherence properties of pseudo-thermal light source (PTS) as a function of laser spot size at the rotating diffuser plate. The laser spot size is varied by translating microscope objective lens towards or away from the diffuser plate. The longitudinal spatial coherence length, which governs the axial resolution of the coherence microscope, is found to be minimum for the beam spot size of $3.5 \mathrm{~mm}$ at the diffuser plate. The axial resolution of the system is found to be equal to $\sim 13 \mu \mathrm{m}$ at $3.5 \mathrm{~mm}$ beam spot size. The change in the axial resolution of the system is confirmed by performing the experiments on standard gauge blocks of height difference of $15 \mu \mathrm{m}$ by varying the spot size at the diffuser plate. Thus, by appropriately choosing the beam spot size at the diffuser plane, any monochromatic laser light source can be utilized to obtain high axial-resolution irrespective of the source's temporal coherence length. It can provide speckle free tomographic images of multilayered biological specimens with large penetration depth. In addition, PTS avoids the use of any chromatic aberration corrected optics and dispersion-compensation mechanism unlike conventional setups.
\end{abstract}

1. Introduction

Coherence properties of laser light source made a great impact in the field of various optical interferometry, digital holography and optical coherence microscopy/tomography (OCM/OCT) [1-4]. Lasers being highly temporally coherent (TC) in nature can generate interference pattern quickly in optical interference microscopy systems, which further leads to the measurement of various parameters like phase/height map, refractive index and dry mass density of the biological specimens [5]. However, it reduces the interferogram's quality significantly due to the formation of speckle noise, coherent noise and parasitic fringe formation[5, 6]. Moreover, high TC length of the lasers makes them unfit in OCM and OCT applications. In OCM/OCT, generally low temporal coherence light sources such as halogen lamp, LEDs and broadband lasers are employed to obtain interference pattern only from a selected planes of multilayered objects [1,2]. Employment of low TC light source is advantageous for coherent artifacts free phase and optically sectioned images of the biological specimens, however, these sources severely suffer from the problems of chromatic aberration and dispersion $[7,8]$.

Recently, a number of studies have been carried out to synthesize a spatially extended and temporally coherent light source named pseudo-thermal light source (PTS) to overcome aforementioned issues with the conventional light sources $[3,7,9,10]$. PTS can be generated by employing different approaches such as rotating diffuser, vibrating multiple multi-mode fiber bundle (MMFB) and electro-active-polymer rotational micro-optic diffuser [7, 11-13]. The synthesized light source carries the advantages of both high TC and low TC light sources. This makes PTS an attractive alternative of conventional light sources for various industrial and biological applications. PTS have been successfully implemented in optical profilometry, quantitative phase microscopy and OCM/OCT techniques to improve their performance $[3,7,9]$.

In the past, a lot of theoretical and experimental work has been done to understand and study the coherence properties of PTS. The coherence properties, especially the longitudinal spatial coherence (LSC) properties, of PTS as a function of source size, numerical aperture (NA) of microscope objective and wavelength have been studied [9, 
$11,12,14,15]$. Previously, the source size at the input port of the OCM system is varied and observed that it significantly affects the LSC length/axial resolution of the pseudo-thermal light source [11]. The axial resolution of OCM system was found to be high for larger source size at the input port of OCM system or MMFB output port. As the large number of pixels (N: 6400) is present in the MMFB over its 4 mm diameter, therefore, optimally uniform light coupling into each pixel of MMFB is difficult to obtain. Thus, even for a maximum source size at the input port of OCM system, it is needed to understand and optimize the source size at the diffuser plane/input of MMFB for achieving high axial resolution in the OCM system.

Therefore, it is important to understand the effect of source size at the RD plane or input port of MMFB on the LSC length of OCM system systematically. In the present work, PTS is synthesized by passing a high TC and high spatial coherence (SC) light beam (He-Ne laser @ 632nm) through a rotating diffuser followed by a MMFB of effective diameter of $\sim 4 \mathrm{~mm}$. Linnik interferometer based OCM system is utilized to measure the LSC length of PTS as a function of beam spot size at the diffuser plane. It is observed that the axial resolution (half of LSC length) depends on the beam spot size at diffuser plane and found to be equal to $\sim 13 \mu \mathrm{m}$ for $3.5 \mathrm{~mm}$ beam size. A $10 \times(0.3$ NA) microscope objective is utilized to perform all experimentation. To further support our experimental findings, experiments are conducted on standard gauge blocks (height difference $15 \mu \mathrm{m}$ ). The change in the visibility of interference pattern on one of the gauge block is measured while keeping approximately constant visibility at the other one. The visibility of the interference pattern at left side gauge block is decreased as the beam spot size at the diffuser plane is increased. Thus, high axial resolution and wide field of view (FOV) can be achieved in OCM/OCT system with high TC $\left(\mathrm{l}_{\mathrm{c}} \sim 15 \mathrm{~cm}\right)$ laser. This is contrary to the conventional OCM/OCT system, which utilizes low TC light sources to obtain high axial resolution.

\section{Materials and methods}

\subsection{Coherence theory of optical fields}

In the coherence theory of optical fields, Wiener-Khintchine theorem is used for the determination of TC function $[16,17]$ which describes the fixed or constant phase relationship, i.e., correlation between light vibrations at two different moments of time [14]. Analogous to the Wiener-Khintchine theorem [17, 18], the generalized van-CittertZernike theorem $[14,16,19]$ relates LSC function to the angular frequency structure. Figure 1 illustrates the ray diagram exhibiting the concept to determine the longitudinal spatial coherence function of the extended light source $S(u, v) . \theta_{x}, \theta_{y}$ and $\theta_{z}$ are the angles between the beam propagation direction and $x, y$, and $z$ axis, respectively, as illustrated in Fig. 1.

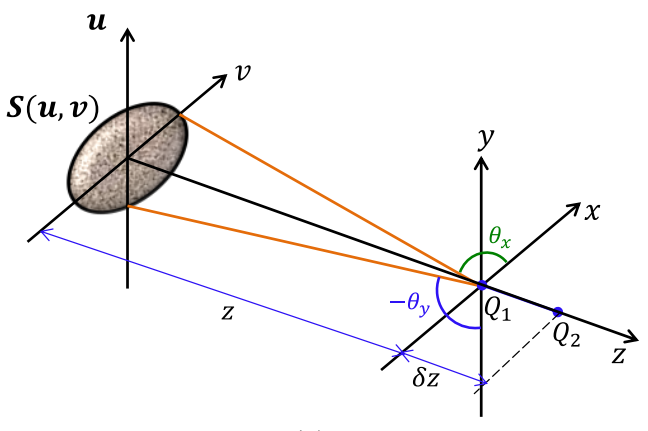

(a)

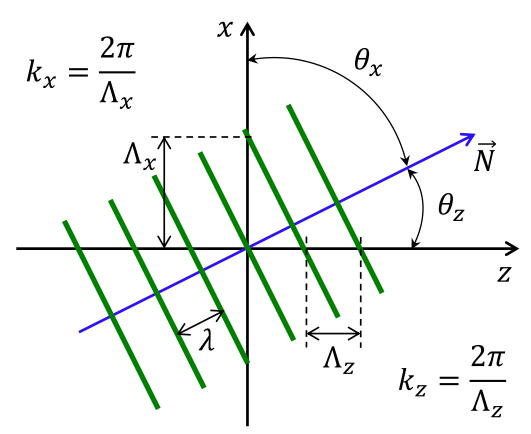

(b)

Fig. 1. (a) Manifestation to determine longitudinal spatial coherence function of an extended source. $S(u, v)$ : is an extended light source. (b) Spatial periods and spatial frequencies of a plane wave propagating along the direction $\overrightarrow{\mathrm{N}}$.

The generalized van-Cittert-Zernike theorem states that LSC function ' $\Gamma(\delta z, \Delta t=0)$ ' and source angular frequency spectrum form Fourier transform pairs. The LSC function is defined as follows:

$$
\Gamma(\delta \mathrm{z}, \Delta \mathrm{t}=0)=\int_{-\infty}^{\infty} \mathrm{S}\left(\mathrm{k}_{\mathrm{z}}\right) \exp \left(\mathrm{ik}_{\mathrm{z}} \delta \mathrm{z}\right) \mathrm{dk}_{\mathrm{z}}
$$


where $\Gamma(\delta \mathrm{z}, \Delta \mathrm{t}=0)$ is the LSC function and $\mathrm{S}\left(\mathrm{k}_{\mathrm{z}}\right)$ is the angular frequency spectrum of the light source, $\delta \mathrm{z}$ $\left(=z_{1}-z_{2}\right)$ is the separation between spatial points $Q_{1}\left(z_{1}\right)$ and $Q_{2}\left(z_{2}\right)$ situated in two different observation planes along the propagation direction of field, and $\mathrm{k}_{\mathrm{z}}$ is the longitudinal spatial frequency[16].

$$
\mathrm{k}_{\mathrm{z}}=\frac{2 \pi}{\Lambda_{\mathrm{z}}}=\frac{2 \pi}{\lambda} \cos \theta_{\mathrm{z}}
$$

where $\Lambda_{\mathrm{z}}$ is the spatial period along the z-direction. $\theta_{\mathrm{z}}$ is the angle between the direction of propagating field and $\mathrm{z}$ axis and $\lambda$ is the wavelength of light source.

From Eq. (1), the longitudinal coherence length can be defined as:

$$
\mathrm{L}_{\mathrm{c}}=\frac{2 \pi}{\Delta \mathrm{k}_{\mathrm{z}}}
$$

where $\Delta \mathrm{k}_{\mathrm{z}}$ is the longitudinal spatial frequency range.

The general expression of the coherence length $\left(\mathrm{L}_{c}\right)$ which depends on both the angular frequency and temporal frequency spectrum of the light source, as follows[16]:

$$
\mathrm{L}_{\mathrm{c}}=\left[\frac{2 \sin ^{2}\left(\theta_{\mathrm{z} / 2}\right)}{\lambda}+\frac{\Delta \lambda}{\lambda^{2}} \cos ^{2}\left(\theta_{\mathrm{z} / 2}\right)\right]^{-1}
$$

where, $\theta_{\mathrm{z}}$ is half of the angular spectral width, $\lambda$ is the central wavelength, and $\Delta \lambda$ is related to the temporal spectral width of the source.

For a sufficiently narrow temporal frequency spectrum width, second term in the above expression can be neglected and $\mathrm{L}_{\mathrm{c}}$ is determined by the angular frequency spectrum only:

$$
\mathrm{L}_{\mathrm{c}}=\lambda / 2 \sin ^{2}\left(\theta_{\mathrm{z}} / 2\right)
$$

Thus, the axial resolution (i.e., $\mathrm{L}_{\mathrm{c}} / 2$ ) is purely determined by LSC rather than temporal coherence length of the source.

\subsection{Visibility/contrast measurement of the interferogram}

To measure the visibility/contrast of the interferograms, Fourier transform algorithm is employed. The 2D intensity distribution of the interferogram is given by the following expression [20,21]:

$$
f(x, y)=a(x, y)+b(x, y) \cos \left[2 \pi i\left(f_{x} x+f_{y} y\right)+\phi(x, y)\right]
$$

where $\mathrm{a}(\mathrm{x}, \mathrm{y})$ and $\mathrm{b}(\mathrm{x}, \mathrm{y})$ are the background (DC) and the modulation terms, respectively. Spatially-varying phase $\phi(\mathrm{x}, \mathrm{y})$ contains information about the specimen. $\mathrm{f}_{\mathrm{x}}, \mathrm{f}_{\mathrm{y}}$ are the spatial carrier frequencies of interferogram along $\mathrm{x}$ and $\mathrm{y}$ axes, respectively. In practical applications, it is envisaged that $\mathrm{a}(\mathrm{x}, \mathrm{y}), \mathrm{b}(\mathrm{x}, \mathrm{y})$ and $\phi(\mathrm{x}, \mathrm{y})$ are slowly varying functions compared to the variation introduced by the spatial carrier frequencies $f_{x}$, and $f_{y}$.

The above intensity modulation can be rewritten in the following form for convenience

$$
f(x, y)=a(x, y)+c(x, y) \exp \left[2 \pi i\left(f_{x} x+f_{y} y\right)\right]+c^{*}(x, y) \exp \left[-2 \pi i\left(f_{x} x+f_{y} y\right)\right]
$$

where

$$
c(x, y)=b(x, y) \exp (i \phi(x, y))
$$

The Fourier transform of Eq. 7 is given as follows:

$$
\mathrm{F}\left(\xi_{\mathrm{x}}, \xi_{\mathrm{y}}\right)=\mathrm{A}\left(\xi_{\mathrm{x}}, \xi_{\mathrm{y}}\right)+\mathrm{C}\left(\xi_{\mathrm{x}}-\mathrm{f}_{\mathrm{x}}, \xi_{\mathrm{y}}-\mathrm{f}_{\mathrm{y}}\right)+\mathrm{C}^{*}\left(\xi_{\mathrm{x}}+\mathrm{f}_{\mathrm{x}}, \xi_{\mathrm{y}}+\mathrm{f}_{\mathrm{y}}\right)
$$

The term $A\left(\xi_{x}, \xi_{y}\right)$ is simply a background (DC) term at the origin in the Fourier plane. The term $C\left(\xi_{x}-f_{x}, \xi_{y}-\right.$ $f_{y}$ ) corresponds to +1 order term contains information about the object and situated at $\left(+f_{x},+f_{y}\right)$. Similarly, $\mathrm{C}^{*}\left(\xi_{\mathrm{x}}+\mathrm{f}_{\mathrm{x}}, \xi_{\mathrm{y}}+\mathrm{f}_{\mathrm{y}}\right)$ is -1 order term situated at $\left(-\mathrm{f}_{\mathrm{x}},-\mathrm{f}_{\mathrm{y}}\right)$ which carry complex conjugate information about of the specimen. After applying Fourier filtering of zero and +1 order terms present in Eq. 9, the interferogram contrast ' $\mathrm{V}$ ' can be obtained using the following expression: 


$$
\mathrm{V}=\frac{2 \times \text { maximum }\left(\operatorname{abs}\left(\mathrm{C}\left(\xi_{\mathrm{x}}-\mathrm{f}_{\mathrm{x}}, \xi_{\mathrm{y}}-\mathrm{f}_{\mathrm{y}}\right)\right)\right)}{\operatorname{maximum}\left(\operatorname{abs}\left(\mathrm{A}\left(\xi_{\mathrm{x}}, \xi_{\mathrm{y}}\right)\right)\right)}
$$

\subsection{Experimental details of PTS based OCM/OCT}

The experimental scheme of the developed system is based on the principle of Linnik based interference microscopy system (Fig. 2a). The light beam emanating from He-Ne laser (lc $=15 \mathrm{~cm}$ ) is made incident onto the microscope objective $\mathrm{MO}_{1}(10 \times / 0.25 \mathrm{NA})$ which illuminates the rotating diffuser (Edmund optics: part \# 62-620; grit size 220) with a diverging beam at $4 \mathrm{~cm}$ away from the center. The speed of the rotating diffuser (RD) at the irradiated point is $8 \mathrm{~cm} / \mathrm{s} . \mathrm{MO}_{1}$ is mounted onto a single axis (x-axis) translation stage to control the size of the beam spot at the diffuser plane. The scattered photons from the RD are directly coupled into a multiple multi-mode fiber bundle (MMFB) placed at $\sim 1 \mathrm{~mm}$ distance from the diffuser plane to maximize the number of coupled photons into MMFB. The active diameter and length of MMFB is $4 \mathrm{~mm}$ and $1.8 \mathrm{~m}$, respectively. It contains large number $(\mathrm{N} \sim 6400$ ) of multimode fibers having core diameter of $\sim 50 \mu \mathrm{m}$ and numerical aperture of 0.65 (Wuzhou Aokace Technology Co., Ltd).

The RD followed by MMFB generates temporally varying speckle field and eventually reduces the speckle contrast significantly. The output port of MMFB acts as a spatially extended purely monochromatic light source namely pseudo thermal light source (PTS). Thus, generates a temporally high and partially spatially coherent light source having short LSC length, which depends on the spatial extent of the light source. Here, the maximum source size is decided by the active diameter of the MMFB. At the same time, it also depends on the illumination at the input port of the MMFB. When scattered photons at the output of RD are incident on a MMFB then the coupling of light is not uniform into all fibers of the MMFB as depicted in Fig. 2b. Thus, each fiber of MMFB does not contribute to decide the LSC length of the PTS even for the full opening of the active diameter of MMFB. This overall increases the LSC length or poor the axial resolution of PTS based OCM/OCT system.

Since, the coupling of scattered photons into individual fiber of MMFB depends on the k-vectors present at the output of RD. Therefore, when the distance between the focal plane of the objective lens and diffuser input surface is increased, the diameter of the input beam interacting with the spatially positioned scattering centers at the diffuser is also increased. Thus, the larger beam size at RD plane increases the number of spatially situated scattering sites. The input beam diameter of $3.5 \mathrm{~mm}$ at the diffuser input facet generates a range of k-vectors at each spatial location of the pixel present in the fiber bundle. The maximum beam diameter of $3.5 \mathrm{~mm}$ at the input facet of the diffuser is decided by the active core diameter $(\sim 4 \mathrm{~mm})$ of MMFB. The input beam of size of $3.5 \mathrm{~mm}$ generates a forward scattered beam of size of greater than or equal to $4 \mathrm{~mm}$. Thus, each pixel of MMFB would contain sufficient range of $\mathrm{k}$-vectors within the NA of individual multimode fiber. This would guide almost equal number of photons into each multimode fiber of MMFB and generates approximately uniform intensity at the output of MMFB. Figs. $2 b$ and $2 c$ depict the effect of input light beam diameter at the output facet intensity uniformity of MMFB and coupling efficiency into each pixel of MMFB. The large beam diameter enables the uniform coupling of the input light beam into each pixel of MMFB and leads to the reduction of LSC length. 


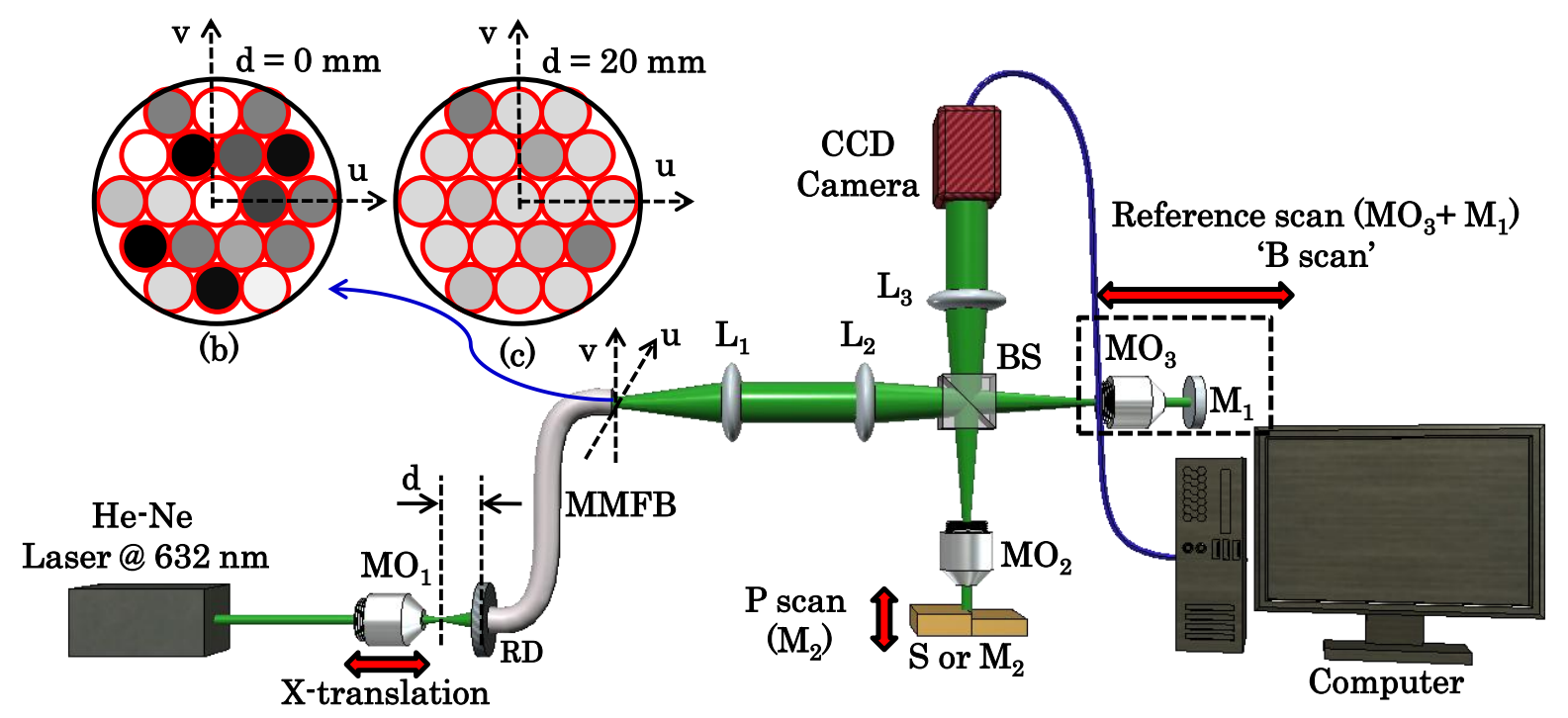

(a)

Fig. 2. (a) Schematic diagram of the spatial coherence gated FF-OCT and OCM system. MO1-3: Microscope objectives; BS: Beam splitter; $\mathrm{L}_{1-3}$ : Lenses; RD: Rotating diffuser; MMFB: Multiple multi-mode fiber bundle; S: sample; $\mathrm{M}_{1-2:}$ Mirrors and CCD: Charge coupled device. $d$ is the distance between focal position of MO1 and RD plane. (b, c) Intensity distribution at the output of MMFB at $\mathrm{d}=0$ and $20 \mathrm{~mm}$, respectively. The different gray values represent the amount of intensity at each fiber of MMFB.

The output port of MMFB is attached with the input port of Linnik based interference microscopy system (Fig. 2a). The combination of lenses $\mathrm{L}_{1}$ and $\mathrm{L}_{2}$ relay the source image (fibers of MMFB) at the back focal plane of the microscope objective $\mathrm{MO}_{2}(10 \times, 0.3 \mathrm{NA})$ to achieve uniform illumination at the sample plane. The back aperture of the objective lens $\left(\mathrm{MO}_{2}\right)$ would limit the range of k-vectors coming from the MMFB output and further control the angle ' $\theta_{\mathrm{z}}$ ' of the light beam after $\mathrm{MO}_{2}$ from its optical axis (Eq. 5). Thus, only those k-vectors will contribute to decide the LSC length of OCM system, which are not blocked by the objective lens. The detailed explanation can be found in Ref. [11]. The beam splitter BS splits the light beam into two; one directed towards the sample (S) and the other one towards reference mirror $\left(M_{1}\right)$. Both light beams reflected back from $S$ (or $\left.M_{2}\right)$ and $M_{1}$ recombine in order to generate the interference pattern at the same beam splitter plane, which is projected at the camera plane with the help of $\mathrm{L}_{3}$. The reference mirror $\mathrm{M}_{1}$ controls the angle between the object and the reference beam, i.e., the fringe width of the interferogram. The reference mirror $M_{1}$ is kept at a particular angle for which high fringe density without aliasing effect is observed at the charge coupled device (CCD) plane. The CCD camera [Lumenera Infinity 2, $1392 \times 1024$ pixels, pixel size: 4:65×4:65 $\mu \mathrm{m}^{2}$ ] is utilized for all interferometric recordings. Subsequently, Fourier transform method [20] is employed for the measurement of visibility/contrast of the recorded interferograms. The image post processing took $\sim 1-2 \mathrm{~s}$.

\subsection{Experimental result with gauge block sample}

The standard gauge blocks are utilized to understand the influence of distance 'd' on the LSC length of PTS based OCM/OCT system. Two gauge blocks of height difference of $15 \mu \mathrm{m}$ are placed side by side as depicted in Fig. 3a. The height difference between the gauge blocks is confirmed by employing white light interference microscopy (WLIM) system. For the illumination, tungsten halogen lamp at $560 \mathrm{~nm}$ central wavelength is used as a light source. The FWHM of the light source is around $300 \mathrm{~nm}$ varies from $460 \mathrm{~nm}$ to $760 \mathrm{~nm}$. The details of WLIM can be found in refs. [22, 23]. Since, the temporal coherence length of white light is around $1.6 \mu \mathrm{m}$, therefore, the height difference of $15 \mu \mathrm{m}$ between the gauge blocks is confirmed by shifting the white light fringe from the left side gauge block to right side gauge block with an accuracy of $0.8 \mu \mathrm{m}$ (half of the coherence length of white light). This shifting of white light fringe is done by vertically translating the sample stage in a step of $1 \mu \mathrm{m}$. Figures $3 \mathrm{~b}$ and $3 \mathrm{c}$ illustrate the recorded white light interference patterns on the left side and right side gauge block, respectively. The white light fringe width variation on the left and right side gauge blocks could be due to the different angles of gauge blocks as the fringe spacing depends on the angle between object and reference beams. These gauge blocks are further utilized to understand the influence of distance 'd' on the LSC length of PTS. 

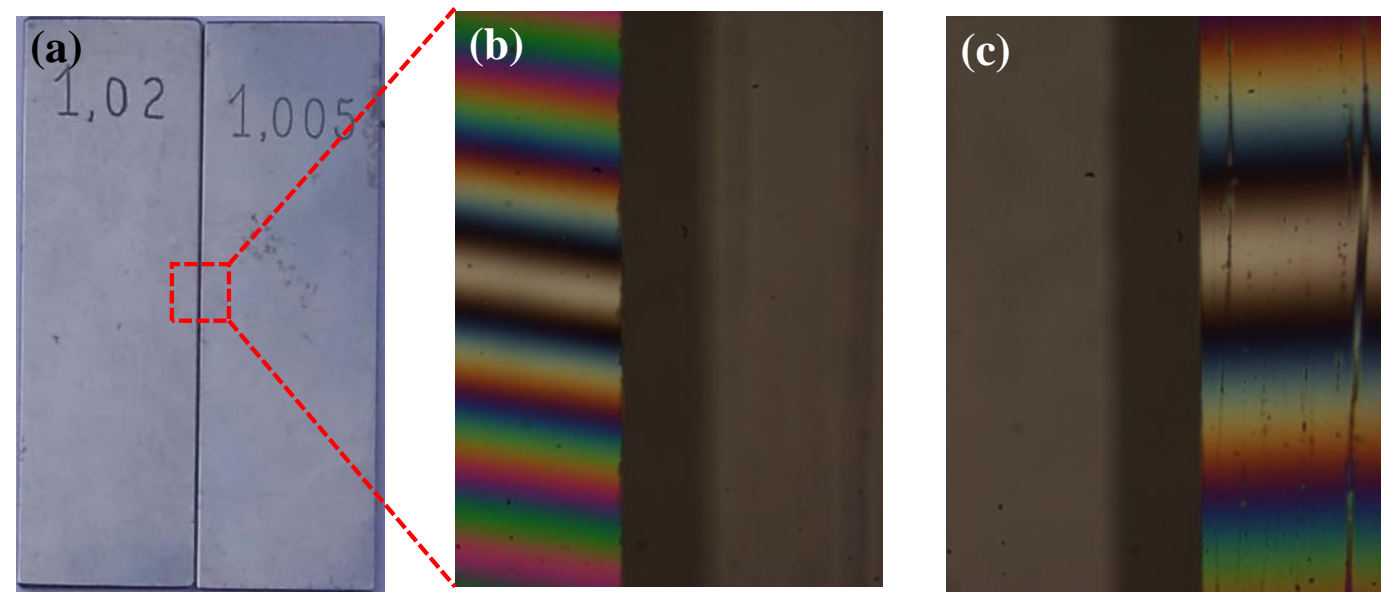

Fig. 3. (a) Standard gauge blocks of height difference of $15 \mu \mathrm{m}$. (b, c) White light interferograms on the left and right side gauge block. The shifting of white light fringe is carried out by vertically translating the sample stage in a step of $1 \mu \mathrm{m}$.

\section{Results and discussion}

\subsection{Longitudinal spatial coherence measurement}

To understand the influence of beam spot size at the RD plate on the LSC length of PTS, $\mathrm{MO}_{1}$ is translated towards or away from RD plane as illustrated in Fig. 2a. A Linnik interference microscopy system is used to realize LSC function experimentally as a function of distance between the focal position of $\mathrm{MO}_{1}$ and $\mathrm{RD}$ plane. For the measurement of LSC length or axial resolution (i.e., LSC/2), a flat mirror $\mathrm{M}_{2}$ (Fig. 1a) as a test sample is placed under the interference microscope and scanned vertically in a step of $1 \mu \mathrm{m}$ from $-\mathrm{z}$ to $+\mathrm{z}$ (P scan) to sequentially acquire a series of interferograms. The visibility/contrast of the series of interferograms are then measured by employing Eq. 10 and plotted as a function of vertical positions of $\mathrm{M}_{2}$ as depicted in Fig. 4a. The blue solid curve illustrated in Fig. 4a exhibits the LSC function of pseudo thermal light source. It is observed that the fringe visibility of interferograms reduces as $\mathrm{M}_{2}$ moves away from the focal position of microscope objective $\mathrm{MO}_{2}$. The FWHM of the LSC function thus obtained which provides information about the axial resolution ' $\Delta \mathrm{z}$ ' and subsequently LSC length $(=2 \Delta \mathrm{z})$ of PTS.
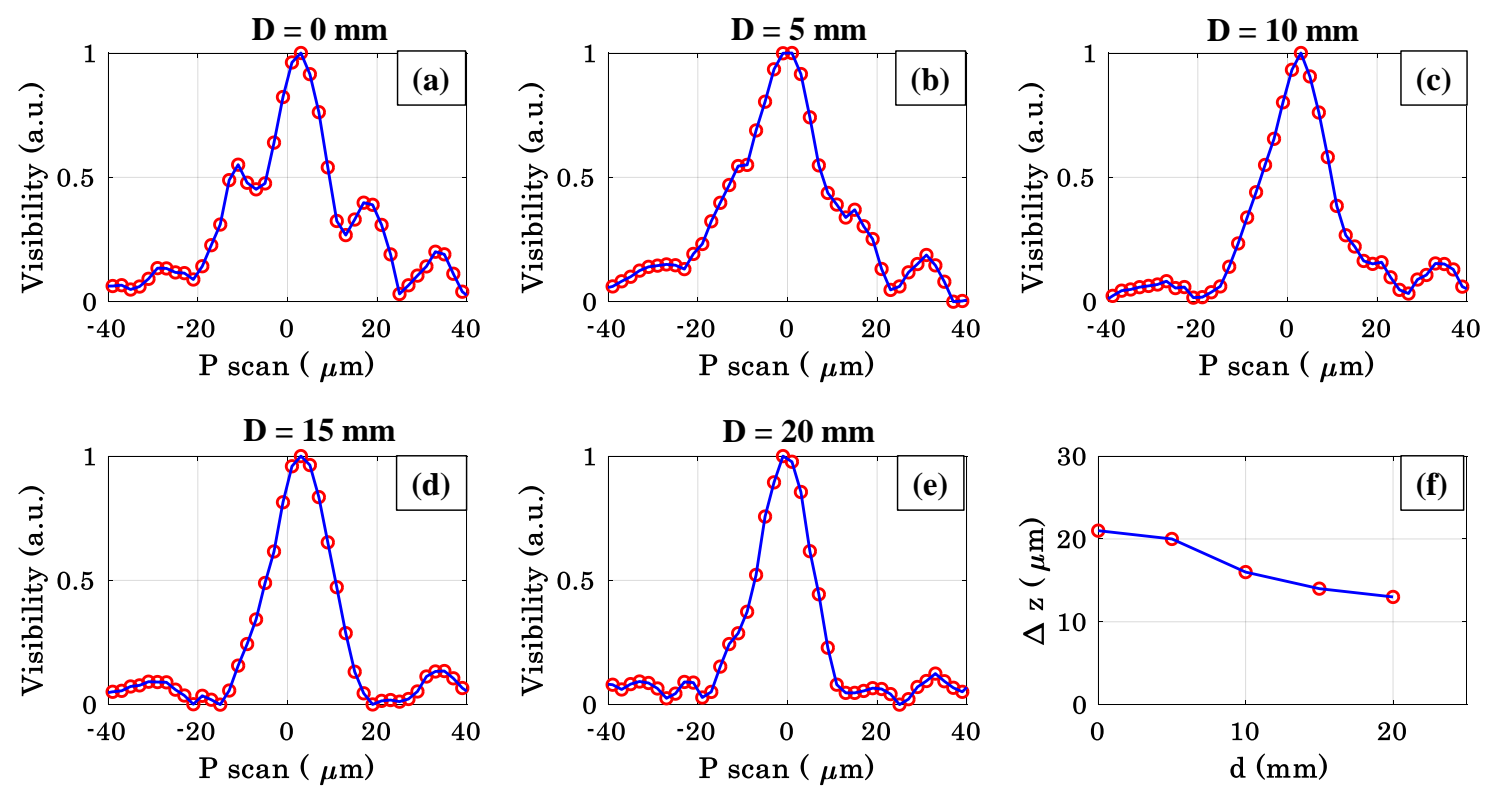

Fig. 4. The LC functions of extended monochromatic light source synthesized from a high coherent He-Ne laser (lc $15 \mathrm{~cm}$ ) as a function of distances ' $\mathrm{D}$ ' (a- e) $0-20 \mathrm{~mm}$ in a step of $5 \mathrm{~mm}$, respectively. (f) Variation of the axial resolution of PTS as a function of distance ' $d$ '. 
Figures $4 \mathrm{a}-4 \mathrm{e}$ present the normalized visibility curves of PTS for the values of $\mathrm{d}$ equal to $0,5,10,15,20 \mathrm{~mm}$, respectively. These values of $d$ correspond to the beam spot sizes of $0.9,1.5,2.4,2.8$ and $3.5 \mathrm{~mm}$ at the diffuser plane. To measure the FWHM of the visibility curves accurately, first, the minimum value of the visibility is subtracted from the visibility data and then normalized by dividing from its maximum value. In other words, the offset is removed from each visibility data and then normalized for the accurate estimation of FHWM as shown in Fig. 4. This leads to the precise measurement of LSC length and axial resolution of the OCM microscope. The maximum beam spot size of $3.5 \mathrm{~mm}$ at the diffuser input is found to be sufficient for the effective utilization of all the fibers present in MMFB. We performed experiments even for the beam size greater than $3.5 \mathrm{~mm}$, however, the significant effect on LSC length/axial resolution is not observed. This could be due to the matching or overfilling of the scattered field with MMFB active diameter.

A slight asymmetry in the measured visibility curves could be due to slight misalignment in the light beam path. The FWHM of each visibility curves are then calculated to obtain axial resolution of the system as given in Table 1. It can be clearly seen from Table 1 that the axial resolution is found to be equal to $\sim 13 \mu \mathrm{m}$ for the distance of $20 \mathrm{~mm}$. The reason behind obtaining the short LSC length for the large separation of $20 \mathrm{~mm}$ between focal plane of $\mathrm{MO}_{1}$ and RD plane has been explained in the materials and method section.

Figure $4 \mathrm{f}$ represents the variation of axial resolution as a function of distance between the focal plane of $\mathrm{MO}_{1}$ and $\mathrm{RD}$ plane. It is worth noting that axial resolution decreases to $13 \mu \mathrm{m}$ at the value of ' $\mathrm{d}$ ' equal to $20 \mathrm{~mm}$. Thus, high axial resolution can be obtained with a monochromatic laser irrespective of its high TC length. The LSC length, i.e., axial resolution, of the system can be further improved with the employment of high NA imaging objective lens $\left(\mathrm{MO}_{2}\right)$ as it widen the angular frequency spectrum of the light source $[11,14,24]$. The use of such light sources are advantageous as it does not require any dispersion compensation and chromatic aberration corrected optics, which are otherwise mandatory in case of broadband light sources [8]. In Table 1, we have listed the outcomes of Fig. 4.

Table 1. The axial resolution ' $\Delta \mathrm{z}$ ' of the PTS synthesized from He-Ne laser as a function of distance 'D' between focal position of M01 and RD plane.

\begin{tabular}{|l|l|l|l|}
\hline S. No. & $\begin{array}{c}\text { 'D' } \\
(\mathbf{m m})\end{array}$ & $\begin{array}{c}\text { Beam spot size at } \\
\text { diffuser plane } \\
(\mathbf{m m})\end{array}$ & $\boldsymbol{\Delta \mathbf { z } ( \boldsymbol { \mu m } )}$ \\
\hline 1. & 0 & 0.9 & 21 \\
\hline 2. & 5 & 1.5 & 20 \\
\hline 3. & 10 & 2.4 & 16 \\
\hline 4. & 15 & 2.8 & 14 \\
\hline 5. & 20 & 3.5 & 13 \\
\hline
\end{tabular}

\subsection{Effect on the fringe visibility of gauge blocks}

To further confirm the reduction in the LSC length of PTS as the distance between $\mathrm{MO}_{1}$ and $\mathrm{RD}$ plane is increased, experiments are conducted on standard gauge blocks. Two standard gauge blocks with height difference of $15 \mu \mathrm{m}$ are placed side by side as illustrated in Fig. 3a. Their height difference is confirmed by WLIM as presented in Figs. $3 \mathrm{~b}$ and $3 \mathrm{c}$. Figures $5 \mathrm{a}-5 \mathrm{e}$ illustrate the recorded interferograms of the same gauge blocks for d equal to $0,5,10,15$, $20 \mathrm{~mm}$, respectively, by employing the experimental setup presented in Fig. 2a. The interferograms of the gauge blocks are recorded with approximately constant visibility at the right-side gauge block for all sets of experiments. It is clearly visualized from Figs. $5 \mathrm{a}-5 \mathrm{e}$ that the fringe contrast is reduced on the left side gauge block as the distance between focal position of $\mathrm{MO}_{1}$ and $\mathrm{RD}$ plane is increased. It means that the axial resolution of the PTS based OCM/OCT system can be increased by appropriately choosing the beam spot size at the RD plane, which is controlled by translating $\mathrm{MO}_{1}$ towards or away from $\mathrm{RD}$ plane. The experiments are conducted with $10 \times(0.3 \mathrm{NA})$ objective lens for the present study.

Table 2 illustrates the calculated values of the fringe contrast at the left and the right side gauge blocks corresponding to Figs. 5a - 5e. The visibility of the interferograms of the standard gauge blocks are normalized with respect to the right side gauge block. It can be clearly seen that the visibility of the interferogram at the left side gauge block is reduced to 0.44 for the value of d equal to $20 \mathrm{~mm}$. 

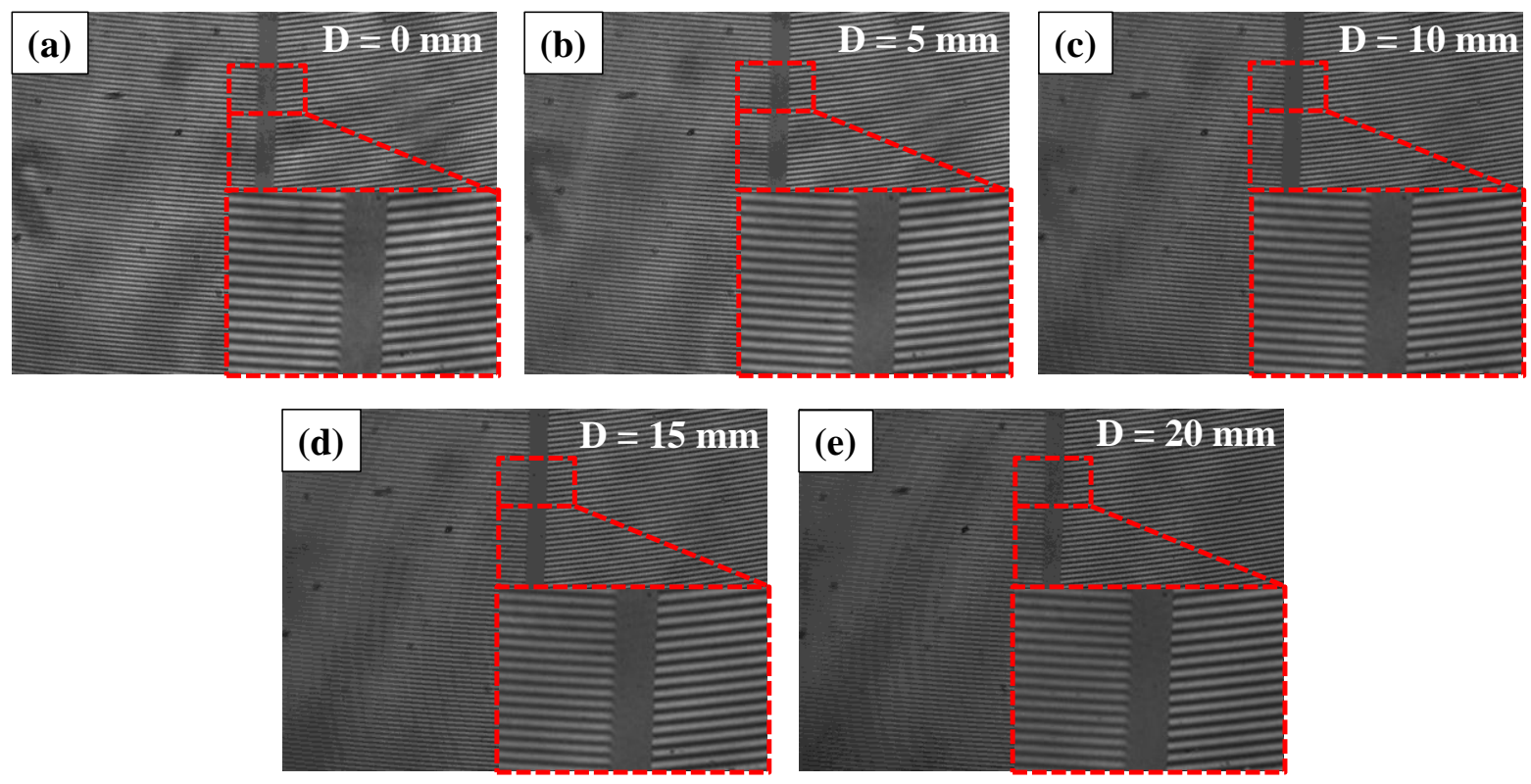

Fig. 5. Visibility of the interferograms at the left and the right side gauge block as a function of distance ' $D$ '. (a - e) Recorded interferograms of two standard gauge blocks having $15 \mu \mathrm{m}$ height difference corresponding to the values of $d$ equal to $0,5,10,15,20 \mathrm{~mm}$. The visibility is kept constant visibility at the right side gauge block.

Table 2. Variation of visibility of the interferograms at the left and the right side gauge block as a function of distance ' $\mathrm{D}$ '. The visibility at the right side gauge block is kept approximately constant to compare the change in the fringe contrast at the left side gauge block.

\begin{tabular}{|c|l|l|l|l|l|}
\hline S. No. & $\begin{array}{c}\text { D } \\
\text { (mm) }\end{array}$ & \multicolumn{2}{|c|}{ Visibility (a.u.) } & \multicolumn{2}{c|}{$\begin{array}{c}\text { Norm. visibility w.r.t. } \\
\text { right gauge block (a.u.) }\end{array}$} \\
\cline { 3 - 6 } & & $\begin{array}{c}\text { Left gauge } \\
\text { block }\end{array}$ & $\begin{array}{l}\text { Right gauge } \\
\text { block }\end{array}$ & $\begin{array}{c}\text { Left gauge } \\
\text { block }\end{array}$ & $\begin{array}{c}\text { Right } \\
\text { gauge } \\
\text { block }\end{array}$ \\
\hline 1. & 0 & 0.2089 & 0.3058 & 0.6832 & 1 \\
\hline 2. & 5 & 0.1931 & 0.3018 & 0.6399 & 1 \\
\hline 3. & 10 & 0.1787 & 0.3063 & 0.5836 & 1 \\
\hline 4. & 15 & 0.1545 & 0.2991 & 0.5166 & 1 \\
\hline 5. & 20 & 0.1355 & 0.3065 & 0.4421 & 1 \\
\hline
\end{tabular}

\section{Conclusion}

In conclusion, proposed system provides high resolution optical sectioning of the multilayered samples by implementing a low LSC of PTS based OCM/OCT system, which is otherwise not possible with the direct laser. This is contrary to the principle of conventional OCM/OCT system, which utilized low TC length sources like halogen lamp or LEDs to perform high resolution sectioning of the biological specimens. The influence of source size at the $\mathrm{RD}$ plane by translating the microscope objective $\mathrm{MO}_{1}$ on the axial resolution of PTS is systematically studied. The axial resolution of $\sim 13 \mu \mathrm{m}$ is observed for PTS at spot size of $3.5 \mathrm{~mm}$, which is otherwise impossible to obtain with the monochromatic laser like He-Ne of TC length of $15 \mathrm{~cm}$. Thus, high resolution sectioning of the specimen can be performed with the employment of a sufficiently wide angular spectrum, i.e., low LSC length PTS irrespective of the TC length of the parent laser. In addition, any appropriate laser (wavelength falling in the biological window) compatible to the biological samples can be used for the synthesis of PTS and further implemented for high resolution optical sectioning with large penetration depth. Further, PTS generates speckle free images of the biological specimens. It can provide significant enhancement in the measurement sensitivity compared to the broadband laser based existing OCM/OCT systems. In future, PTS will be implemented for performing high axial resolution sectioning of the biological samples with large penetration depth. This type of light source does not require any chromatic aberration corrected optics and dispersion compensation mechanism unlike broadband light source like halogen lamp based OCM/OCT systems. 
Funding. The authors are thankful to Department of Atomic Energy (DAE), Board of Research in Nuclear Sciences (BRNS) for financial grant no. 34/14/07/BRNS.

\section{References}

1. W. Drexler and J. G. Fujimoto, Optical coherence tomography: technology and applications (Springer Science \& Business Media, 2008).

2. H. M. Subhash, "Full-field and single-shot full-field optical coherence tomography: A novel technique for biomedical imaging applications," Adv. Opt. Technol. 2012(2012).

3. A. Ahmad, and Dalip Singh Mehta, "Quantitative Phase Microscopy and Tomography with Spatially Incoherent Light," in Advances in Optics: Reviews (2018), pp. 487-511.

4. D. S. Mehta, V. Srivastava, S. Nandy, A. Ahmad, and V. Dubey, "Full-field optical coherence tomography and microscopy using spatially incoherent monochromatic light," in Handbook of Full-Field Optical Coherence Microscopy (Pan Stanford, 2016), pp. 379-414.

5. G. Popescu, Quantitative phase imaging of cells and tissues (McGraw Hill Professional, 2011).

6. A. Ahmad, V. Dubey, V. Singh, A. Butola, T. Joshi, and D. S. Mehta, "Reduction of spatial phase noise in the laser based digital holographic microscopy for the quantitative phase measurement of biological cells," in European Conference on Biomedical Optics, (Optical Society of America, 2017), 104140H.

7. J. Rosen and M. Takeda, "Longitudinal spatial coherence applied for surface profilometry," Appl. Opt. 39, 4107-4111 (2000).

8. L. Vabre, A. Dubois, and A. C. Boccara, "Thermal-light full-field optical coherence tomography," Opt. Lett. 27, 530-532 (2002).

9. A. Ahmad, Vishesh Dubey, Gyanendra Singh, Veena Singh, and Dalip Singh Mehta, "Quantitative phase imaging of biological cells using spatially low and temporally high coherent light source," Opt. Lett. 41, 1554-1557 (2016).

10.M. H. Pavliček Pavel, Zhihui Duan, and Mitsuo Takeda, "Spatial coherence profilometry on tilted surfaces," Appl. Opt. 48, H40-H47 (2009).

11.A. Ahmad, Tanmoy Mahanty, Vishesh Dubey, Ankit Butola, Balpreet Singh Ahluwalia, and Dalip Singh Mehta, "Effect on the longitudinal coherence properties of a pseudothermal light source as a function of source size and temporal coherence," Opt. Lett. 44, 1817-1820 (2019).

12.A. Ahmad, V. Srivastava, V. Dubey, and D. Mehta, "Ultra-short longitudinal spatial coherence length of laser light with the combined effect of spatial, angular, and temporal diversity," Appl. Phys. Lett. 106, 093701 (2015).

13.H. Farrokhi, Thazhe Madam Rohith, Jeeranan Boonruangkan, Seunghwoi Han, Hyunwoong Kim, Seung-Woo Kim, and Young-Jin Kim, "High-brightness laser imaging with tunable speckle reduction enabled by electroactive micro-optic diffusers." ." Sci. Rep. 7(2017).

14.I. Abdulhalim, "Spatial and temporal coherence effects in interference microscopy and full-field optical coherence tomography," Ann. Phys. 524, 787-804 (2012).

15.V. Ryabukho, Dmitry Lyakin, and Mikhail Lobachev, "Longitudinal pure spatial coherence of a light field with wide frequency and angular spectra." " Opt. Lett. 30, 224-226 (2005).

16.V. Ryabukho, D. Lyakin, A. Grebenyuk, and S. Klykov, "Wiener-Khintchin theorem for spatial coherence of optical wave field," Journal of Optics 15, 025405 (2013).

17.L. Mandel and E. Wolf, Optical coherence and quantum optics (Cambridge university press, 1995).

18.J. W. Goodman, Statistical optics (John Wiley \& Sons, 2015).

19.I. Abdulhalim, "Competence between spatial and temporal coherence in full field optical coherence tomography and interference microscopy," Journal of Optics A: Pure and Applied Optics 8, 952 (2006).

20.M. Takeda, Hideki Ina, and Seiji Kobayashi, "Fourier-transform method of fringe-pattern analysis for computer-based topography and interferometry," J. Opt. Soc. Am. A 72, 156-160 (1982).

21.A. Ahmad, Vishesh Dubey, Vijay Raj Singh, Jean-Claude Tinguely, Cristina Ionica Øie, Deanna L. Wolfson, Dalip Singh Mehta, Peter TC So, and Balpreet Singh Ahluwalia, "Quantitative phase microscopy of red blood cells during planar trapping and propulsion," Lab Chip 18, 3025-3036 (2018).

22.V. Dubey, Singh, V., Ahmad, A., Singh, G. and Mehta, D.S., "White light phase shifting interferometry and color fringe analysis for the detection of contaminants in water," in Proc. SPIE, 2016), 97181F. 
23.A. Ahmad, Anand Kumar, Vishesh Dubey, Ankit Butola, Balpreet Singh Ahluwalia, and Dalip Singh Mehta, "Characterization of color cross-talk of CCD detectors and its influence in multispectral quantitative phase imaging," Opt. Express 27, 4572-4589 (2019).

24.I. Abdulhalim, "Competence between spatial and temporal coherence in full field optical coherence tomography and interference microscopy." " J. Opt. A: Pure Appl. Opt. 8(2006). 